\title{
The Noise Dipole Source Prediction of Far-Field Acoustic Pressure for Marine Propeller using Inverse Method
}

\author{
Bagheri MR ${ }^{1}$, Mehdigholi ${ }^{1 *}$, Seif MS $^{1}$ and Rajabnia $\mathrm{H}^{2}$
}

${ }^{1}$ Department of Mechanical Engineering, Centre of Excellence in Hydrodynamic and Dynamic of Marine Vehicles, Sharif University of Technology, Iran ${ }^{2}$ Department of Mechanical Engineering, Zahedan University, Iran

\begin{abstract}
The unsteady rotating force or dipole strength distribution, acting by the fan or propeller on the fluid, is predicted by inverse method. In this method, the far-field acoustic pressures are used in non-cavitating condition. In this paper, the far-field acoustic pressures are obtained from Ffowcs Williams and Hawkings (FW-H) equations using computational fluid dynamic (CFD) in specific hydrophone array and then the unsteady rotating force, acting by the propeller on the fluid, is obtained as the most important sound source in non-cavitating condition. The unsteady rotating forces are extracted using inverse method by analytical code in Matlab. The correct solution is independence to the optimum select of regularization parameter from transfer function; the transfer function represents relationship between the force coefficients and the far-field acoustic pressure. Therefore, the appropriate range of regularization parameter should be choice in order to an ill-conditioned problem from transfer function is solve. The analytical code is solved for different regularization parameters and then the unsteady rotating forces are obtained for three sections on the blade surface. The inverse method could be used for dipole strength distribution calculation as the most important sound source in non-cavitating condition in order to design the noiseless of marine propeller.
\end{abstract}

Keywords: Unsteady rotating force; Far-field acoustic pressure; FW$\mathrm{H}$, Transfer function; CFD

\section{Introduction}

Basically, the blade's noise can be produced in two conditions of cavitations and non cogitation states. In the non-cavitations condition, the dipole sound source is the most important source for generating sound in fluids [1]. The dipole source results from the unsteady force of the rotating blade in a fluid. Aeroacustic equations have been provided by Ffowcs Williams and Hawkings which are the developed form of Lighthill's equations. These relations include the movement in the infinite fluid environment. So, it can be used in driving the noise in fans and blades. There are a lot of researches where sound pressure level of far-field has been investigated by the assist of these equations. In works by Seol et al. in 2002 and 2005 [2,3], Caro et al. [4], Jin-ming et al. [5], Pan et al. [6] and Bagheri et al. [7,8], the SPLs obtained using FW-H Equations. Generally, acoustic pressure can be generated because of three kinds of sources. The first one, which is related to turbulence in fluid, is known as the quadruple source. This source can be influential just in the cases that the Mach number is greater than 0.8 [9-11]. The second source is related to the movement of dipole source and corresponds to the exerted unsteady force by solid surfaces in fluids. This source is known as the loading noise or dipole noise. Dipole source are the most important sources for generating sound in the noncavitation condition. Also, they have a simple translation motion for displacing; hence, predicting of these sources on the different surfaces of a blade is the main goal of this study. The third source is a diffusion of monopole which stems from the effects of volumetric transportation of surfaces and it is known as the thickness noise. As long as the speed in the top of the blade is less than the sound speed, the efficiency of thickness noise is negligible [9-11]. Therefore, in non-cavitating conditions where speed is less than sound speed in water, this source can be eliminated [12]. However, the monopole source is important in cavitation conditions. This is because as cavitations can be considered as a swinging sphere that is alongside with expansion and contraction, it can result in volume injection in the fluid.

Periodic force will lead to the sound production. These forces can be formed in blade passing frequency (BPF) and the multiples of the blade frequencies. There are some researches [2-8] where, although acoustic pressure field in far field has been extracted by solving the flow, dipole sources which are the most important sources of producing sound in non-cavitating conditions have not been considered.

The inverse method can be used for investigating unsteady rotating forces due to the distribution of dipole power created by rotating fan and blades in a fluid. Acoustic pressure measurement in far-field or the tonal noise generated by propellers can be used in this approach. The noises of fans and blades have been in the limelight by researches since the analytical solution had been common by FW-H equations. It has been demonstrated that dipole sources resulted from unsteady forces on blade passing frequency in fluid has the least effect in the Mach number less than 0.8 . Therefore, unsteady pressure over the blade would be equal to the distribution of dipoles in low Mach and non-cavitating conditions [9-11]. The computational fluid dynamics method (CFD) presents the sound pressure levels by the solution of FW-H equations. Thus, the measures of unsteady forces loading by noise in the blade passing frequency can be driven by the prediction of sounds pressure in the hydrophone array with inverse method. On the other hand, the measurement of pressure fluctuations will need a wide range of experimental approaches such as applying piezo plastic sensors or other kinds of accurate pressure transducers.

Lee et al. [13], provided an inverse method to reconstruction of pressure distribution over the blade in propagated sound field. Their

*Corresponding author: Mehdigholi H, Department of Mechanical Engineering Centre of Excellence in Hydrodynamic and Dynamic of Marine Vehicles, Sharif University of Technology, Iran, Tel: +98 216601 3126; E-mail: mehdi@sharif.edu

Received September 28, 2017; Accepted October 23, 2017; Published October 28, 2017

Citation: Bagheri MR, Mehdigholi H, Seif MS, Rajabnia H (2017) The Noise Dipole Source Prediction of Far-Field Acoustic Pressure for Marine Propeller using Inverse Method. J Appl Mech Eng 6: 290. doi: 10.4172/2168-9873.1000290

Copyright: (c) 2017 Bagheri MR, et al. This is an open-access article distributed under the terms of the Creative Commons Attribution License, which permits unrestricted use, distribution, and reproduction in any medium, provided the original author and source are credited. 
researches were based on inverse solution of $\mathrm{FW}-\mathrm{H}$ integral equations. $\mathrm{Lu}$ et al. [14], suggested a model of air acoustic of interactions in addition, a rotor and a stator according to integral equations of sound. Also, there are some other researches who worked on the interactions of a plate in a jet stream [15-17]. One of the most recent studies has been done by Trabelsi et al. [18] where unsteady rotating forces in a fan have been derived using inverse method.

In this paper, the unsteady forces will be derived which caused by the rotation of the blade and will lead to noise production in the blade passing frequency. First of all, the far-field acoustic pressure in an appropriate hydrophone array will be drived by $\mathrm{FW}-\mathrm{H}$ equations in a CFD method and then the forces of rotating blade as the most important source of producing sound in non-cavitating conditions in three sections will be drived by an analytical code using the indirect inverse method.

\section{Prediction of the Unsteady Rotating Force by Inverse Method}

\section{Indirect driving of inverse equations}

There is a lot of theoretical investigation on calculating the acoustic pressure as a function of fluctuating forces caused by the rotation of blade in fluid $[19,20]$. It is assumed that these forces as the most important source of producing noise can be measured or drived through a mathematical approach by solving equation of propagated sound of Morse or FW-H equations [21]. These forces have been defined as the term of the dipole source in the Helmholtz and Lighthill equations which are the main equations for the acoustic pressure field. The basic equation for evaluating the propagated sound is the Lighthill equations that have been achieved from the combination of the continuous and momentum equations. The Lighthill equations can be introduced as the Equation (1) [22].

$$
\nabla^{2} p^{\prime}-\frac{1}{c^{2}} \frac{\partial^{2} p^{\prime}}{\partial t^{2}}=\dot{q}+\nabla \cdot f+\frac{\partial^{2} \hat{o}_{i j}}{\partial x_{i} \partial x_{j}}
$$

The left side of the Lighthill equations is related to sound propagation and the right side introduces the sound production sources. The first term in the right side that affects cavitations condition is associated with the mass or volume injection into the fluid and is entitled as the monopole source. The second term that is related to the pressure difference is the loaded force in fluid. Also, the third term that can be neglected in low speeds is the Lighthill stress tensor.

The function of these equations, which have been used in order to drive the sound pressure field of propeller in far-field in this paper, can be used to predict the acoustic far field from the target, so that, first of all, the source of noise production should be specified by flow solution around the body and then the essential data related to the flow as the sound production source in Lighthill equations will be obtained by introducing the reference level around the body. Finally, the distribution of acoustic pressure from the body can be achieved by solving the integral equation. As it is mentioned before, the dipole source is the main source in non-cavitating conditions. Therefore, the obtained acoustic pressure field from FW-H equations [23] in the non-cavitating conditions will be attributed to the dipole source or unsteady rotating forces in the blade passing frequency that leads to sound production. As the result, the production factors of sound in fluid as the dipole source in any sections of blade can be derived by achieving the acoustic pressure field through the CFD method and applying it in formulating the inverse method. A reference coordinate

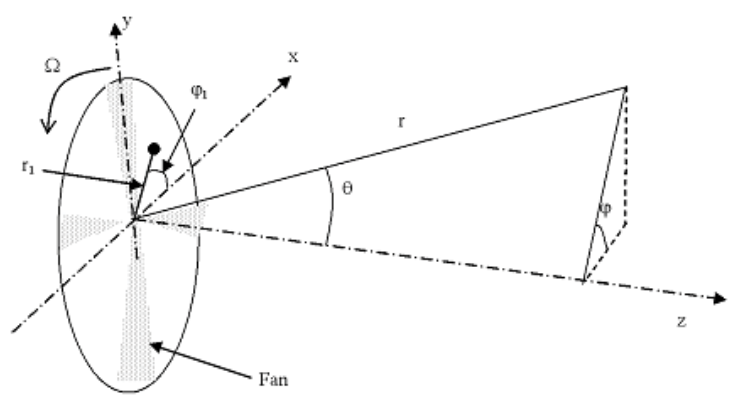

Figure 1: Coordinate system for source and receiver.

system would be needed to introduce the hydrophone array and the locations of loaded forces. This coordinate system shows in Figure 1.

As it can be seen from Figure 1, polar coordinates have been chosen to indicate a point on the surface of the blade. In fact, this point will represent a section of the blade and unsteady perpendicular loading forces on the blade should be driven at this point as the source of sound production. Furthermore, it would be a need for another coordinate system for acoustic pressure field to use the inverse method. So, the spherical coordinate system will be used in order to indicate the characteristics of a point in acoustic field. The main reason for using inverse model is driving the loading unsteady forces on the unit area in section $(r, \varphi)$ in the BPF.

The axial force per unit surface is shown by $F_{z}$ which is in the direction of $z$. This force can be written as a Fourier series in harmonies of time and circumference as following:

$$
f_{\mathrm{Z}}\left(r_{1},{ }_{1}\right)=\sum_{s=-\infty}^{s=+\infty} \cdot \sum_{q=-\infty}^{q=+\infty} \overline{f_{z}^{0}} \alpha_{\mathrm{s}}\left(r_{1}\right) \beta_{q}\left(r_{1}\right) \mathrm{e}^{i(s B+q)_{1}} \mathrm{e}^{-i s \omega_{1} t}
$$

Where, $\beta_{\mathrm{q}}$ is the Fourier coefficient of circumference harmonies which consists of the unsteady forces caused by propeller rotation in the section of $\left(r_{1}, \varphi_{1}\right)$. Small part of this force which is related to propeller rotation harmonies in low Mach will produce a high level of sound. So, it cannot be negligible. $\alpha_{\mathrm{s}}$ is the Fourier coefficient of time harmonies. Following to that, $\omega_{1}$ is the angular velocity, $B$ is the number of blades, $\mathrm{s}$ is counter of blade's harmonies and $\mathrm{Q}$ can be deemed as the number of harmonies or the number of points on a cross-section as the integrated band. The acoustic pressure can be obtained by calculating the integration of rotating unsteady forces in the cross-section $\mathrm{A}$ of the blade as the following:

$$
p(t ; r, \varphi, \theta)=\int_{A}^{0} \int_{\mathrm{z}}\left(t ; r_{1}, \varphi_{1}\right) g_{1 \mathrm{z}}\left(t ; r_{1}, \varphi_{1} ; r, \varphi, \theta\right) r_{1} d r_{1} d \varphi_{1}
$$

Where, $g_{1 z}$ is the sound field from a point force in the direction of $z$ in the location of $\left(\mathrm{r}_{1}, \varphi_{1}\right)$. $\mathrm{g}_{1 \mathrm{z}}$ can be introduced as the following:

$$
g_{1 z}=-i k \cos \theta \frac{\mathrm{e}^{i k r}}{4 \partial r} \sum_{m=-\infty}^{+\infty} i^{m} \mathrm{~J}_{m}\left(k r_{1} \sin \theta\right) \mathrm{e}^{i m\left(\varphi-\varphi_{1}\right)} \mathrm{e}^{-i \omega t}
$$

Here, $k=\omega / c$ is the acoustic wave number, $c$ is the sound velocity, and $J_{\mathrm{m}}$ is the cylindrical Bessel function of order $\mathrm{m}$. In order to achieve a relation between acoustic pressure field of far field and blade unsteady rotating forces in fluid, Equations (2) and (4) should be replaced in (3). Then, using the orthogonal relations and expanding the integral to $r_{1}$, Equation (5) can be shown as the following: 


$$
\begin{aligned}
P_{s j} & =-\frac{i k_{1} \cos \theta_{j}}{4 \pi r} \sum_{q=q_{\min }}^{q=q_{\max }} i^{s B+q} \mathrm{e}^{i s k_{1} r_{j}} \mathrm{e}^{i(s B+q) \varphi_{j}} \\
& \times \sum_{i=1}^{l} s \overline{f_{z}^{0}}\left(r_{1 i}\right) \alpha_{s}\left(r_{1 i}\right) \beta_{q}\left(r_{1 i}\right) \mathrm{J}_{s B+q}\left(s k_{1} r_{1 i} \sin \theta\right) 2 \pi r_{1 i} \Delta r_{1}
\end{aligned}
$$

Which can be compacted as Equation (6):

$$
P_{\mathrm{sj}}=\sum_{L} H_{\mathrm{sjL}} f_{\mathrm{sL}}
$$

Indexes $i$ and $q$ are related to the cross-sections and circumference harmonies, respectively, and it is shown as the function of $L=(i, q)$. Also, $f_{\mathrm{sl}}$ is the vector of sound source that represents the characteristics of the propagated dipole source power in the radial position of $i$ in time and circumference harmonies. In addition,

$$
\begin{aligned}
& H_{\mathrm{sjL}}=-\frac{i s k_{1} \cos \theta_{j}}{2 r_{j}} i^{s B+q} \mathrm{e}^{i s k_{1} r_{j}} \mathrm{e}^{i(s B+q) \varphi_{j}} \\
& \mathrm{~J}_{s B+q}\left(s k_{1} r_{1 i} \sin \theta\right)
\end{aligned}
$$

$r_{1 i} \Delta r_{1}$ is a transition function that can relate the power of sound source $f_{\mathrm{sl}}$ to the propagated sound field. Equation (6) is a linear system shown in the form of matrix in Equation (8):

$$
\{P\}=[H]\{f\}
$$

$P_{\mathrm{s}}$ is a vector of measured acoustic pressure in far-field in the location of $j$ and $f_{\mathrm{s}}$ is a vector of unsteady rotating axial forces per unit surface loaded by the blade in fluid. $\mathrm{H}_{\mathrm{s}}$ is a transient function between multiples of forces and acoustic pressure of far-field which can be gained by Equation (7). All of these measures are defined for $s$ of the BPF. So, Equation (8) can be rewritten as Equation (9):

$$
\left(\begin{array}{c}
P_{s}^{1} \\
\vdots \\
P_{s}^{j}
\end{array}\right)=\left[\begin{array}{ccc}
H_{1 q_{\min }} & \cdots & H_{1 q_{\max }} \\
\vdots & \ddots & \vdots \\
H_{j q_{\min }} & \cdots & H_{j q_{\max }}
\end{array}\right]\left(\begin{array}{c}
f_{s-q_{\min }} \\
\vdots \\
f_{s-q_{\max }}
\end{array}\right)
$$

Where, $p_{\mathrm{sj}}$ is the Fourier coefficient of measured acoustic pressure in $j$ hydrophones, $f_{s-q \max }$ is the Fourier coefficient of rotating unsteady force loaded by the blade in fluid, and $\mathrm{H}_{\mathrm{iq}}$ are the transfer matrix elements. The aim of inverse method would be the calculation of the vector of sound source, $f_{\mathrm{s}}$, from the measured acoustic pressure data in the far-field, $p_{s}$. The measured acoustic pressure in the far-field is defined as $p_{\mathrm{s}}$ which is calculated through CFD method in this paper. The vector $f_{\mathrm{s}}$ can be obtained by the approach of Nelson and Yon for estimating the power of dipole source in the inverse method [21].

$$
f_{s}=H_{s}^{+} \hat{P}_{s}
$$

Here, $H_{s}^{+}=\left[H_{s}^{H} H_{s}\right]^{-1} H_{s}^{H}$ is a reconstructed matrix of $H_{s}$. If $\left[H_{s}^{H} H_{s}\right]$ is a positive-definite matrix, the problem will have unique answer. In addition, if the number of hydrophones ( $j$ ) is equal to the number of sound source arrays on the desired section, $\mathrm{i}$, the answer can be written as $f_{s}=H_{s}^{-1} \hat{P}_{s}$. If the number of hydrophones is less than the number of arrays of sound source vector, answer won't exist. Whatever the rows of transfer matrix are more, the matrix will be well-posed. Therefore, many numbers of hydrophones in the sound array will be specified to increase the number of rows of the transfer matrix and then, acoustic pressure field can be obtained by solving FW-H equations in CFD. In the case of less $k$, matrix $H_{\mathrm{s}}$ will be well-conditioned and small errors will not lead to change in the answer of the force vector in the pressure field. However, in large $k$, the problem will be ill-conditioned. This is because; small changes in $P_{\mathrm{s}}$ will result in large errors in the answer. In order to avoid making large changes in $H_{s}$, and having an ill-conditioned problem, stabilization method is applied.

A regulator parameter, $\beta$, should be considered to solve Equation (10). In the following section, it is explained that how this parameter will be obtained. Ultimately, the answer will be as Equation (11), which is used to obtain the force in each section of the blade.

$$
f_{s 0}=\left[H_{s}^{H} H_{s}+\beta I\right]^{-1} H_{s}^{H} \hat{P}_{s}
$$

\section{Selecting the appropriate regulator coefficient}

The most important thing to have a well-conditioned problem would be a suitable regulator, $\beta$. One of the applicable approaches is the L-curve criterion [21]. The L-curve criterion consists of drawing norm 2 of vector $\mathrm{f}$ of the well-conditioned answer in Equation (11) to norm 2 of remaining vector $\left\|\hat{P}_{s}-P_{s}\right\|$ in the logarithmic scale in different amount of $\beta . \hat{P}_{S}$ is the acoustic pressure gained from CFD approach and $P s$ is the pressure gained directly by Equation (8). The general L-curve has been shown in Figure 2.

There are two main zones in the Figure 2. Between these two zones, a suitable coefficient can be found where the remaining of $\left\|\hat{P}_{s}-P_{s}\right\|$ would be small and the regulated answer can have a small norm of $\left\|f_{s}\right\|$. A suitable $\beta$ can be accompanied by decreasing the condition number and following to that a correct answer is obtained. In the other words, some parameters such the number of hydrophones and their positions which are depended on $\theta$ and $\varphi$ angels should be selected properly. In this paper, suitable parameters are used which have been suggested by Presezniak et al. [23].

\section{Modeling the Numerical Analysis and Presenting Re- sults in CFD}

\section{Developing the grid and presenting hydrodynamics results}

In this paper, a five-blade propeller model is used which has $A_{E} / A_{0}=0.7$ and $A_{E} / A_{0}=0.7$. This model was designed at the Center of Excellence in Hydrodynamics and Dynamics of Marine Vehicles (CEHDMV) and is a research model with high application at CEHDMV. Figure 3 shows several information and quantities, such as the geometries, surface grids on the blade and hub surfaces, the computational domains of the model, and the boundary conditions. The solution of the Unsteady RANS equations for utilizing the ReNormalization Group (RNG) $k-\varepsilon$ turbulence model and the FWH sound equation is performed by the CFD. The RNG $k-\varepsilon$ model is based on the standard $\left\|\hat{P}_{s}-P_{s}\right\|$ model but has many advantages [23]. Type of the grid, size of the meshes, and quality are the main

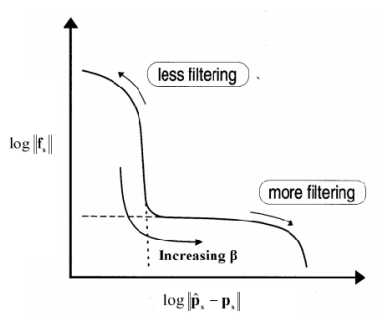

Figure 2: The generic form of the L-curve [21]. 
contributing factors in the accuracy of numerical simulation of any geometry, since their compositions affect the convergence/divergence of the solution to a great extent. Here, convective terms are discretized using the second order accurate upwind scheme, while the velocitypressure coupling and the overall solution procedure are based on the Semi-Implicit Method for Pressure Linked Equations-Consistent (SIMPLEC) type. The blade surface is meshed with triangles grids, while the regions around the root, tip and blade edges are meshed with smaller triangles, i.e. with sides of approximately $0.001 \mathrm{D}$. The remaining region in the domain is then filled with hexahedron cells.

We also considered zones, named rotating zones, which contained the flow around the propeller, and stationary zones which contained the flow around the moving zone. A cylindrical shape is assumed for the rotating zone, with a diameter of $1.3 \mathrm{D}$ and a length of $1.3 \mathrm{~L}$, where $\mathrm{L}$ is the length of propeller hub. The rotating zone is solved via Moving Reference Frame (MRF). The inlet is situated in $4 \mathrm{D}$ distance in the upstream, while the outlet is located at $10 \mathrm{D}$ downstream and the outer boundary is at $5 \mathrm{D}$ from the shaft axis. In order to simulate the flow around the rotating propeller where the inlet boundary is located, we had imposed the velocity components for a uniform stream with a given inflow speed. At the blade and hub surface, a wall condition had imposed, while a wall boundary condition along with constant pressure conditions are imposed at the lateral and outlet boundaries, respectively.

It is important to keep the cell thickness along the body thinner than the boundary layer. The value of coefficient $y^{+}$was the main criterion for setting the mesh resolution. The coefficient should be in a range of $30<y^{+}<500$ in order to model properly the turbulent boundary layer and obtain correct pressure distributions on the propeller blade surfaces for the $\left\|\hat{P}_{s}-P_{s}\right\|$ model. The $y^{+}$value along the propeller surface was around +27 to 110 (Figure 4 ).

Variety kinds of grid system have been examined numerically in the research. As the answer should be independent from the number of grids, the results of Thrust and Torque coefficient have been

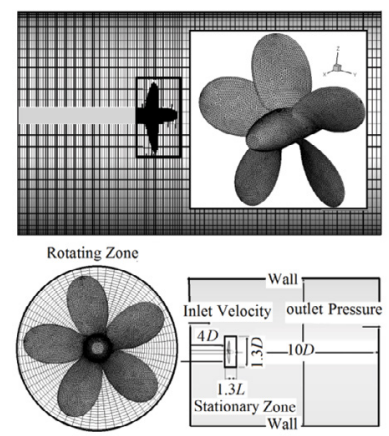

Figure 3: Grids of model, computational domain and boundary conditions.

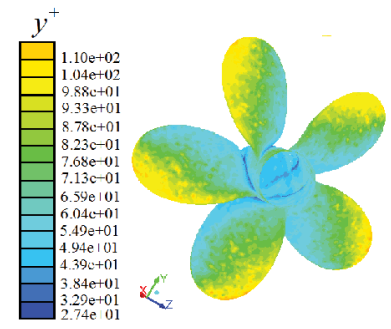

Figure 4: Distribution of $y+$ on the blade surface.

\begin{tabular}{|c|c|c|c|c|}
\hline \multicolumn{5}{|c|}{$\mathrm{J}=0.4$} \\
\hline Variables & $\mathrm{K}_{\mathrm{T}}$ & $\mathbf{K}_{\mathrm{Q}}$ & Error $\mathrm{K}_{\mathrm{T}}(\%)$ & Error $\mathrm{K}_{\mathrm{Q}}(\%)$ \\
\hline Experiment & 0.3454 & 0.05298 & - & - \\
\hline Grid 1 & 0.3101 & 0.04905 & 10.22 & 6.53 \\
\hline Grid 2 & 0.3396 & 0.05174 & 1.67 & 2.34 \\
\hline Grid 3 & 0.3391 & 0.05168 & 1.82 & 2.45 \\
\hline \multicolumn{5}{|c|}{$\mathrm{J}=0.6$} \\
\hline Variables & $\mathrm{K}_{\mathrm{T}}$ & $\mathbf{K}_{\mathrm{Q}}$ & Error $\mathrm{K}_{\mathrm{T}}(\%)$ & Error $\mathrm{K}_{\mathrm{Q}}(\%)$ \\
\hline Experiment & 0.2495 & 0.04088 & - & - \\
\hline Grid 1 & 0.2203 & 0.03793 & 11.70 & 7.21 \\
\hline Grid 2 & 0.2359 & 0.03897 & 5.45 & 4.67 \\
\hline Grid 3 & 0.2348 & 0.03834 & 5.89 & 6.21 \\
\hline \multicolumn{5}{|c|}{$\mathrm{J}=0.8$} \\
\hline Variables & $\mathbf{K}_{\mathrm{T}}$ & $\mathbf{K}_{\mathbf{Q}}$ & Error $\mathrm{K}_{\mathrm{T}}(\%)$ & Error $\mathrm{K}_{\mathrm{Q}}(\%)$ \\
\hline Experiment & 0.1451 & 0.0270 & - & - \\
\hline Grid 1 & 0.1359 & 0.0238 & 6.34 & 11.85 \\
\hline Grid 2 & 0.1447 & 0.0259 & 1.89 & 4.07 \\
\hline Grid 3 & 0.1447 & 0.0259 & 1.89 & 4.07 \\
\hline
\end{tabular}

Table 1: Comparison of grid study and experiment results.

\begin{tabular}{|c|c|c|c|}
\hline Number of Hydrophones & $\boldsymbol{r}^{\boldsymbol{m}}(\mathbf{m}) \mathbf{0}$ & $\theta$ (rad) & $\varphi \mathbf{i}(\mathbf{i}=\mathbf{1} . .64)(\mathbf{r a d})$ \\
\hline 64 & 1.5 & 1.5533 & $i \times \pi / 32$ \\
\hline 32 & 1.5 & 1.5533 & $i \times \pi / 16$ \\
\hline
\end{tabular}

Table 2: Positions of 64 hydrophones.

\begin{tabular}{|c|c|c|c|c|c|c|}
\hline \multicolumn{2}{|c|}{$\mathbf{0 . 9} \mathbf{R}, \mathbf{s}=\mathbf{1}$} & \multicolumn{2}{|c|}{$\mathbf{0 . 7 ~ R , ~ s = 1 ~}$} & \multicolumn{2}{c|}{$\mathbf{0 . 5} \mathbf{R}, \mathbf{s = 1}$} & \multirow{2}{*}{ row } \\
\hline $\mathbf{k}$ & $\boldsymbol{\beta}$ & $\mathbf{k}$ & $\boldsymbol{\beta}$ & $\mathbf{k}$ & $\boldsymbol{\beta}$ & \\
\hline $2 \times 10^{14}$ & $10^{-20}$ & $1.21 \times 10^{14}$ & $10^{-20}$ & $6.19 \times 10^{13}$ & $10^{-20}$ & 1 \\
\hline $2 \times 10^{12}$ & $10^{-18}$ & $1.21 \times 10^{12}$ & $10^{-18}$ & $6.19 \times 10^{11}$ & $10^{-18}$ & 2 \\
\hline $2 \times 10^{10}$ & $10^{-16}$ & $1.21 \times 10^{10}$ & $10^{-16}$ & $6.19 \times 10^{9}$ & $10^{-16}$ & 3 \\
\hline $2 \times 10^{8}$ & $10^{-14}$ & $1.21 \times 10^{8}$ & $10^{-14}$ & $6.19 \times 10^{7}$ & $10^{-14}$ & 4 \\
\hline $2 \times 10^{6}$ & $10^{-12}$ & $1.21 \times 10^{6}$ & $10^{-12}$ & $6.19 \times 10^{5}$ & $10^{-12}$ & 5 \\
\hline $2 \times 10^{4}$ & $10^{-10}$ & $1.21 \times 10^{4}$ & $10^{-10}$ & $6.19 \times 10^{3}$ & $10^{-10}$ & 6 \\
\hline $2 \times 10^{2}$ & $10-8$ & $1.21 \times 10^{2}$ & $10^{-8}$ & $6.19 \times 10$ & $10^{-8}$ & 7 \\
\hline 4.01 & $10^{-6}$ & 2.21 & $10^{-6}$ & 1.06 & $10^{-6}$ & 8 \\
\hline
\end{tabular}

Table 3: The different values of condition number and regulator parameter.

investigated for three kinds of grids, 1.5, 3.5 and 4 million grids. The results of grid independency have been shown in Tables 1-3 for three advanced coefficient of $0.4,0.6$ and $j=0.8$. As it can be seen from the tables, the value of Thrust and Torque coefficient can reach around the experimental results by increasing the number of grids from 1.5 to 3.5 million, while by raising the number of grids from 3.5 to 4 , these values will remain consistent. All in all, the suitable number of grids would be 3.5 million and the results will be provided in this condition.

Figure 5 presents the Thrust, Torque and Efficiency coefficients for the various grids in numerical simulation and compared with experimental results. In Figure 5, a good agreement observes between the numerical and experimental results (Table 2).

In this research, unsteady rotating forces are investigated which will lead to produce noise in a marine propeller. According to previous discussion in section 2 , the condition number can be very influential in order to find an appropriate regulator parameter to have a wellconditioned transform matrix. This parameter would have direct effect on the model of geometry and following to that it can affect the surveyed positions. SPLs are presented for the distance $10 \mathrm{R}$ in the propeller rotational plane and in front of the hub. In Figure 6a, two hydrophones on the hub's axis and propeller rotational plane in $10 \mathrm{R}$ from the center of the coordinate system have been shown. As it was represented in Figure 1, $\theta$ and $\varphi$ define the position of hydrophones 
in plate $\mathrm{x}-\mathrm{y}$ and $\mathrm{z}-\mathrm{y}$ respectively. Hydrophones' positions would be effective in the final driven forces in inverse method. So, hydrophones arrangement in the rotating plate of blade for 64 hydrophones should be as they have shown in Figure $6 \mathrm{~b}$.

According to Perszanik's work [23], 64 hydrophones have been deemed in this study where $\theta$ and $\varphi$ from the blade will show their positions as mentioned in Table 2 and Figure $6 \mathrm{~b}$. Also, the results from 16 and 32 hydrophones will represent similar results. Hydrophones distances from the origin, $\mathrm{r}_{\mathrm{f}}$, are equal to $10 \mathrm{R}$ or $0.75 \mathrm{~m}$ for all receivers. Propeller will be the best feature for hydrophones arrangement. Because, this method uses the acoustic pressure of far-field at the BPF to estimate the forces and the most important noise source would be on propeller rotational plane at the BPF. The flow filed should be gained by solving the Navier Stocks equations for 64 hydrophones in Figure $6 \mathrm{~b}$ using CFD. Then, the blade surfaces should be given as the reference level in integral of FW-H formulation. For 64 hydrophones, FW-H formulation should be solved and the history of acoustic pressure field will be obtained.

From Equation (9), pressure values are placed in the frequency domain. Therefore, in order to derive the sound pressure in the frequency domain, Fourier transform series from the values of sound pressure time history will be the solution and then pressure in the frequency domain would be used in Equation (11). An example of time history of sound pressure for hydrophone 1 has been shown in Figure

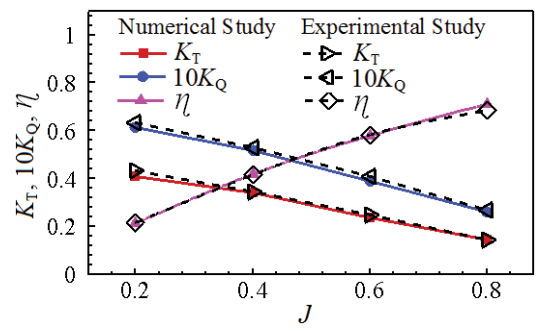

Figure 5: Comparison of numerical simulations with experimental results for trust, torque and efficiency coefficient 3.2. The acoustic pressure results in appropriate hydrophones array.

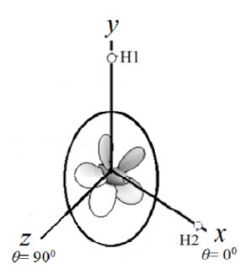

(a)

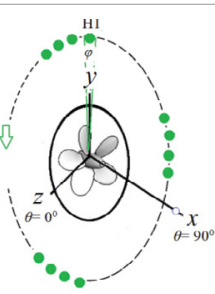

(b)
Figure 6: (a) Two hydrophones on the hub's axis and propeller rotational plane (b) 64 hydrophones positions in propeller rotational plate.

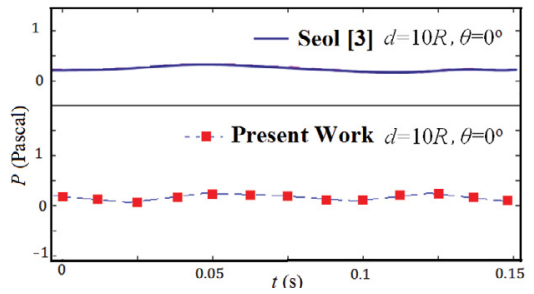

Figure 7: Time history of sound pressure for hydrophone 1.

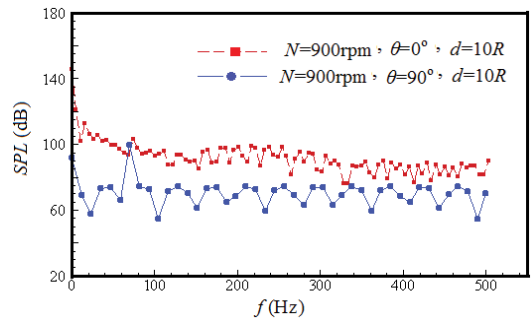

Figure 8: SPLs of hydrophones 1 and 2.

7. In addition, the time history has been derived for other hydrophones and Fourier transform has been carried out for them.

As presented in Figure 7, pressure changes are accompanied by low fluctuations during time in the propeller rotational plane. This is because, pressure changes in non-cavitating conditions are approximately remain constant, thereby the behavior of sound graphs decreases with a constant rate in non-cavitating conditions in frequency domain. However, the range of pressure changes would be large in cavitating conditions, because of its physics and essence that do as a fluctuating spherical large bubble. The results of SPLs of hydrophones 1 and 2 of Figure 6 a have been presented in Figure 8.

Regarding Figure 8, SPL of hydrophone 2 is higher than the first one. Pressure fluctuation in front of the propeller's hub is more than its changes in the propeller rotational plane. Therefore, in the same distance from the origin, sound pressure in front of the hub is higher than that in propeller rotational plane. In Figure 8 , there is a peak in 75 $\mathrm{Hz}$ for the hydrophone placed in $10 \mathrm{R}$ and $\theta=0^{\circ}$ of rotational plane. This peak is related to the first BPF.

\section{Discussion}

\section{Unsteady forces result in 3 cross-sections by inverse method}

As mentioned before, in order to have a well-conditioned answer, an appropriate regulator parameter should be extracted. Having obtained the sound pressure in CFD for 64 hydrophones, the next step would be considering the dipole noise source which is unsteady rotating force.

Since, the accurate answers are subjected to the regulator parameter, the suitable range of $\beta$ for every harmony should be chosen. Then, a sufficient analytical code for every $\beta$ and $\mathrm{k}$ will be provided. Results are presented for $0.5 \mathrm{R}, 0.7 \mathrm{R}$ and $0.9 \mathrm{R}$ sections on blade surface. In the other words, the problem should be solved for $\mathrm{r}_{\mathrm{i}}=0.037, \mathrm{r}_{\mathrm{i}}=0.0525$ and $r_{i}=0.0675 \mathrm{~m}$. In addition, the values of $\mathrm{q}_{\min }$ and $\mathrm{q}_{\text {max }}$ of Equation (9) can be gained by Gerard et al. research [21]. So, $\mathrm{q}_{\min }=-\mathrm{sB}-2$ and $\mathrm{q}_{\max }=-\mathrm{sB}+3$ are considered. Where, $\mathrm{s}$ is a counter of blade's harmonies, $\mathrm{B}$ is the number of blades and $\mathrm{q}$ is the circumference harmonies in the Fourier series of Equation (2).

In this paper, results have been presented for the first harmony, $s=1$. In this situation, the number of $q$ or the elements of force matrix of Equation (9) can be achieved via $\mathrm{Q}=\mathrm{q}_{\max }-\mathrm{q}_{\min }+1$ according to [21]. Therefore, transfer matrix and the force matrix should be $64 \times 6$ and $6 \times$ 1 respectively. The analytical code is written for different domains of $\beta$. Logarithmical values of force can be gained according to the reminding of acoustic pressure for different harmonies. Norm 2 of the vectors $f_{s}$ to Norm 2 of the reminding vector $\left\|\hat{P}_{s}-P_{s}\right\|$ in the logarithmical scale for different values of $\beta$ and for the first harmony in $0.5 \mathrm{R}, 0.7 \mathrm{R}$ and $0.9 \mathrm{R}$ which are shown in Figures 9-11. 


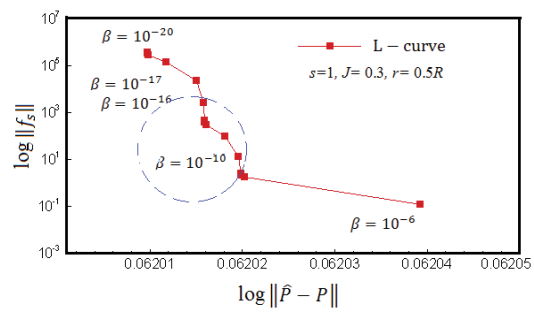

Figure 9: Typical L-curves corresponding to $r=0.5 \mathrm{R}$.

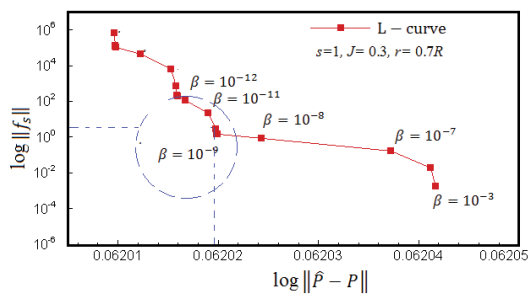

Figure 10: Typical L-curves corresponding to $r=0.7 \mathrm{R}$.

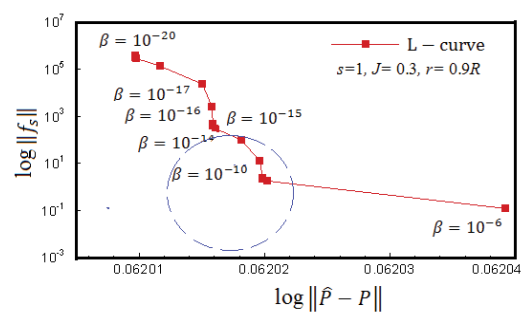

Figure 11: Typical L-curves corresponding to $r=0.9 \mathrm{R}$.

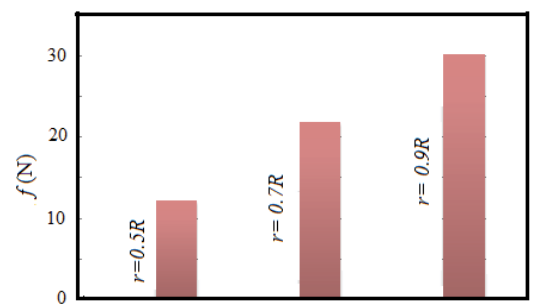

Figure 12: Force average values in three cross-sections of $r=0.5 \mathrm{R}, 0.7 \mathrm{R}$ and $r=0.9$ R.

The suitable value of $\beta$ can be achieved by using the L-curve criterion that has been presented in reference [21]. These values have been depicted in Figures 9-11 with a circular dash line. Sufficient Fourier coefficient and forces have been extracted for the first harmony as the one of the most important ones among cross-sections. So, a suitable range of $\beta$ should be obtained by the $L$-curve criterion for each harmony. Different quantities of condition numbers and $\beta$ have been illustrated in Table 3 . The suitable regulator parameter and condition number can be observed in rows 4 to 7 of this table.

The condition number can play an effective role to select the correct $\beta$ and to calculate the axial perpendicular force on a section of blade. Appropriate values for $\beta$ have been considered $10^{-12}$ to $10^{-7}$. It should be noted that smaller amount of condition number will lead to more desirable answers; however, if condition number is too low, it will result in an unsteady transfer function. Therefore, the appropriate range for condition number has reported as $1.21 \times 10^{-6}$ to $1.21 \times 10^{-4}$.

The inverse method has been applied in order to gain the loading unsteady forces. Force Fourier coefficients in Equation (9) or the amount of $\left(\begin{array}{c}f_{s-q_{\min }} \\ \vdots \\ f_{s-q_{\max }}\end{array}\right)$ can be obtained based on the Equation (11).

The main aim would be gaining and validating the amount of forces from the measured sound pressure obtained in CFD. The inversion of Equation (8) cannot be reachable because the determinant of transfer matrix is equal to 0 . Therefore, the regulator parameter reported by [21] has been applied which leads a well-conditioned transfer matrix. Now; the initial values of force matrix coefficients can be obtained, which are $\left(\begin{array}{c}f_{s-q_{\min }} \\ \vdots \\ f_{s-q_{\max }}\end{array}\right)$. In order to check the coefficients, Acoustic pressure field can be achieved from these values and the initial transfer matrix using Equation (8). These results are compared with the obtained acoustic pressure field results in CFD. If the difference is not acceptable the loop is repeated in order to achieve the appropriate force coefficients. The correct forces average values in three cross-sections of $r=0.5 \mathrm{R}, 0.7 \mathrm{R}$ and $\mathrm{r}=0.9 \mathrm{R}$ shown in Figure 12.

\section{Conclusion}

The unsteady rotating forces of a blade caused by distribution of dipole sources have been investigated in an inverse method. Far field acoustic pressure measurement or the noise on blade passing frequency was applied. Direct inversion of the acoustic pressure relation based on the unsteady rotating force is not well-conditioned. In the other words, the process of inversing the transform matrix which is a relation between the exerted unsteady force from the blade and the measured acoustic pressure in blade frequencies will be confronted problem. So, there should be a technique to have a stable solution. In remodeling the unsteady forces, the issue of inversion will be related to the source and the location of receivers of far field and blade passing frequency. In this paper, the unsteady forces due to the rotation of blade in fluid have been extracted, as the most important source of sound production in non-cavitating conditions using an inversion method. In order to achieve these forces correctly, the inversion of the well-conditioned transfer matrix and an appropriate state number have been derived. The suitable range of regulator parameter and the condition number have been chosen between $10^{-12}$ and $10^{-7}, 1.21 \times 10^{4}$ and $1.21 \times 10^{6}$ respectively. Since the experimental measurement of unsteady forces as the dipole sources will be complicated and piezo sensors would be needed, the inverse method to calculate these forces in non-cavitating conditions is applied. The measurement of these forces in different sections of blade will contribute to design a noiseless propeller. The use of indirect inverse method to reach unsteady forces as the dipole source of sound in a marine propeller is the important innovation of this study which has been conducted for the first time for this kind of propeller and can lead to design a noiseless propeller.

\section{References}

1. Carlton JS (1994) Marine propellers and propulsion. Butterworth Heinemann London, UK.

2. Seol H, Jung B, Suh JC, Lee S (2002) Prediction of non-cavitating underwater propeller noise. J Sound Vibration 257: 130-157.

3. Seol H, Suh JC, Lee S (2005) Development of hybrid method for the prediction of underwater propeller noise. J Sound Vibration 288: 345-360.

4. Caro S, Sandboge R, Iyer I, Nishio Y (2007) Presentation of a CAA formulation 
Citation: Bagheri MR, Mehdigholi H, Seif MS, Rajabnia H (2017) The Noise Dipole Source Prediction of Far-Field Acoustic Pressure for Marine Propeller using Inverse Method. J Appl Mech Eng 6: 290. doi: 10.4172/2168-9873.1000290

Page 7 of 7

based on LightHill's analogy for fan noise. Conference on Fan Noise Lyon. pp. 17-19.

5. Jin-ming Y, Ying X, Fang L, Zhang-Zhi W (2012) Numerical prediction of blade frequency noise of cavitating propeller. J Hydrodynamics 24: 371-377.

6. Pan Y, Zhang HX (2013) Numerical prediction of marine propeller noise in nonuniform in flow. J China Ocean Eng 27: 33-42.

7. Bagheri MR, Seif MS, Mehdigholi $H(2013)$ Hydrodynamic and acoustic analysis of underwater propellers by numerical method. J Maritime Technology 9: 1-14.

8. Bagheri MR, Seif MS, Mehdigholi $H$ (2014) An analysis of hydrodynamics and noise behavior for submerged propeller in various conditions by experimental and numerical methods. J Modares Mech Eng 14: 15-25.

9. Goldstein ME (1976) Aeroacoustics, McGraw-Hill International Book Company, USA. pp. 293.

10. Wright SE (1969) Sound radiation from a lifting rotor generated by asymmetric disk loading. J Sound Vibration 9: 223-240.

11. Tyler JM, Sofrin TG (1994) Axial flow compressor noise studies. Transaction of the Society of Automotive Engineers 70: 309-332.

12. Neise W (1992) Review of fan noise generation mechanisms and contro methods. Fan Noise 92: 45-56.

13. Lee XD, Zhou S (1996) Spatial transformation of the discrete sound field from a propeller. American Institute of Aeronautics J 34: 1097-1102.

14. Lue J, Li XD (1998) An inverse aeroacoustics problem on rotor wake/stator interaction. J Sound Vibration 254: 219-229.

15. Grace SP, Atassi HM (1996) Inverse aeroacoustics problem for a streamlined body, part 1: Basic formulation. American Institute of Aeronautics and Astronautics J 34: 2233-2240.

16. Grace SP, Atassi HM (1996) Inverse aeroacoustics problem for a streamlined body, part 2: Basic formulation. American Institute of Aeronautics and Astronautics J 34: 2241-2246.

17. Wood TV, Grace SM (2000) Inverse aeroacoustics problem for a rectangular wing. American Institute of Aeronautics J 38: 203-210.

18. Trabelsi H, Abid M, Taktak M, Fakhfakh T, Haddar M (2014) Reconstruction of the unsteady rotating forces of fan's blade from far-field sound pressure. Applied Acoustics.

19. Lowson MV (1968) Theoretical analysis of compressor noise. J Acoustical Society of America 47: 371-385.

20. Grad A, Berry A, Masson P (2005) Reconstruction of aeroacoustics sources from far-field sound pressure. J Sound Vibration 288: 1049-1075.

21. Light Hill MJ (1952) on sound generated aerodynamically: I. General theory. Proc. Royal Society London, UK 211: 564-587.

22. Ffowcs Williams JE, Hawkings DL (1969) Sound generated by turbulence and surfaces in arbitrary motion. Philosophical Transactions of the Royal Society 264: 321-342.

23. Presezniak F, Steenackers G, Guillaume P (2010) Mechanical systems and signal processing. Mechanical Systems and Signal Processing 24: 1682-1692. 